\title{
LE DÉSIR DE LANGUE. LES DIMENSIONS POÉTIQUE ET POLITIQUE DE LA DIGLOSSIE DANS LE ROMAN « LE JARDIN DE VERRE » DE TATIANA ŢÎBULEAC
}

\section{THE DESIRE FOR LANGUAGE. THE POETIC AND POLITICAL DIMENSIONS OF DIGLOSSIA IN THE NOVEL "THE GLASS GARDEN" BY TATIANA TÎ́BULEAC}

\author{
Cristina HERMEZIU \\ Université Alexandru Ioan Cuza de Iași/ Alexandru Ioan Cuza University of Iași, \\ Romania \\ e-mail: hermeziu.cristina@gmail.com
}

\begin{abstract}
Still relevant today via mobility and the multicultural environment as the constitutive elements of our globalized societies, the reflections around "the cumbersome problem of the genius of languages" (Cassin 2016) join and enrich the question of living together and building oneself. In the field of literature or human sciences, the thought of the practice of languages in a situation of diglossia has forged an imaginary which often connects the passage from one language to another to a balance of power and a tension of identity. We propose an analysis of the symbolic values of the dilemma between the Romanian language and the Russian language which is at the heart of the diegesis of the novel Grădina de sticlă [The Glass Garden] by Tatiana Tîbuleac. Published in Romanian by Éditions Cartier de Chișinău and translated into several languages, including French and Spanish, the book was awarded the European Union Prize for Literature in 2019. The fictional world and the language of writing describe a symbolic abyss: the author describes in Romanian the fight with the Russian of a Moldovan orphan who is trying to rebuild herself between the two languages. In a very colorful style, the novel deploys a po(i)etics of "between", which also has a political dimension.
\end{abstract}

Keywords: diglossia; poetics; identity; subjectivation; T,îbuleac.

\section{Prémisses théoriques. Contre le génie des langues}

Lorsque le désir de langue s'avère être la problématique centrale de la diégèse, interroger ses dimensions symboliques semble s'imposer comme une approche analytique appropriée. Pour analyser le devenir du personnage Lastotchka entre deux langues et dégager la po(i)étique du roman Le Jardin 
de verre de Tatiana Țîbuleac, nous convoquons deux théories complémentaires sur le terrain de l'anthropologie linguistique. En premier lieu, la philosophe et philologue Barbara Cassin (2016, 2020) qui, dans plusieurs de ses écrits, entreprend une critique de la perception « relativiste » de la langue comme vision du monde. La philosophe s'insurge contre l'enracinement identitaire (Cassin, 2016: 217) et contre les généralités singularisantes (Cassin, 2016: 201) qui rendent si séduisante la théorie du génie des langues. L'auteur cite et commente, entre autres, Rivarol et son discours De l'universalité de la langue française, primé au concours de l'Académie de Berlin de 1783, où le français est déclaré langue universelle : avec sa syntaxe « incorruptible » (Rivarol, 1998 : 128-129), "sûre, sociale, raisonnable, ce n'est plus la langue française, c'est la langue humaine » (Rivarol, 1998 : 133-134). Pour contrer des pratiques de pouvoir à l'œuvre entre les langues, la philosophe milite pour une attitude d'admiration païenne, anti-idolâtre - sorte de résistance au prestige symbolique d'une langue dominante. Deux mots d'ordre surgissent dans sa pensée autour du rapport de force entre langues, individus et société, à savoir l'impératif d'aller vers "plus d'une langue » et " une langue, ça n'appartient pas » (Cassin, 2016: 220). Dans le sillage de Jacques Derrida (2005) qu'elle convoque, Barbara Cassin résume dans Éloge de la traduction. Compliquer l'universel (2016) :

«[...] la première condition pour savoir qu'on "a" une langue est d'entendre au moins deux. Il faut connaître, ou même seulement approcher, au moins deux langues pour savoir qu'on en parle une, que c'est une langue que l'on parle. À moins de cela, il n'y a pas d'autre, et pas même de soi [...] Derrida, plus déterritorialisé, ajoute que la langue qu'il a "n'est pas la sienne". Car la seconde leçon de la même expérience est que cette langue qu'on a "n'appartient pas". Elle est parlée par d'autres qui en "ont" aussi, ou d'abord, une autre. Dire qu'une langue, ça n'appartient pas, permet de délier langue et peuple, de dénationnaliser la langue, de décloisonner l'espace. »(Cassin, $2016: 218)$

Barbara Cassin fait aussi l'éloge de la traduction comme activité qui "crée le passage entre les langues, entre. Ce faisant, elle se situe d'amblée dans la dimension du politique: il y va de l'articulation d'une pluralité différenciée. Le divers est mis en cuvre dans une pratique du commun. » (Cassin, 2016 : 220-221). Nous retenons aussi le syntagme de «l'étonnement pur » comme suggestion du principe opératoire qui pourrait dissoudre les relations de domination entre des langues qui entrent en contact. Un syntagme opératoire qui nous permet ici d'anticiper un élément significatif de la diégèse du roman Le Jardin de verre. Une Moldave de huit ans est adoptée 
par une Russe, ramasseuse de bouteilles abandonnées dans la rue. L'ouvrage s'ouvre avec une re-naissance que l'entrée pour la première fois dans la ville déclenche dans les yeux émerveillés de l'enfant. L'appropriation du nouveau monde, de la différence, passe par l'étonnement et par l'émerveillement. La véritable naissance de la fillette est dans la langue, sa naissance est à la langue russe.

En seconde lieu, nous nous appuyons sur le terrain de la psychanalyse et ses théories qui mettent en relation la structuration de la subjectivité et le multilinguisme. Ici même, le concept d'«entre les langues» semble être opératoire.

" L'approche des devenir-sujets en situation de contact de langues semble pouvoir opérer plus largement comme un révélateur et un analyseur de la formation de la subjectivité en général. D'une subjectivité-palimpseste, agencement de traits identificatoires divers, en discontinuité, potentiels ou actualisés et non limitatifs. (...) Sujet multistyle, ou sujet multilingue, chaque être parlant s'inscrit dans une logique de l'échange qui est une logique de "traduction". » (Prieur, 2006:111-118)

Employer tantôt une langue, tantôt une autre, en situation de diglossie ou bilinguisme, fantasmer une langue ou une autre est tout à fait légitime, disent les théoriciens, dans le sillage de Deleuze ou de Foucault, cités ici par Jean-Marie Prieur, dans Contact de langues et positions subjectives :

"L',,entre deux langues” n'est pas nécessairement et toujours expérience du malaise et de la douleur, il peut être passage de vie, passage de création (ne fût-ce que celle du mot d'esprit), ,puissance affirmative et affirmée" (Deleuze, 1969 : 344), parce que d'une langue à l'autre peuvent s'inventer des ,ponts verbaux”, des liens, des articulations, ceux d'un réseau associatif opérant à travers mots, phonèmes, assonances ou homophonies. Il peut être création d'un „espace ouvert”, et d'une subjectivité ou d'une trans-subjectivité à vivre dans le passage, la traversée, et qui, dans l'angoisse ou la jouissance du changement de corps et de langue, voit ses frontières questionnées. » (Prieur, $2006: 111-118$ )

Après Cioran, qui a écrit son œuvre d'abord en roumain pour passer définitivement au français en 1946, peut-on concevoir autrement la subjectivation du rapport entre le roumain et le français que sous le signe d'un pattern antagoniste ? Dans l'œuvre de Cioran, on trouve de nombreuses occurrences où l'écrivain roumain d'expression française s'attarde sur le rapport entre ses deux langues d'écriture qui opposent le lyrisme à la lucidité, la liberté à la discipline, l'impétuosité à la sobriété, l'excessif à la retenue. La 
question subsidiaire qui se pose vise à dégager, dans l'imaginaire littéraire de la diglossie, une figure particulière qui implique la langue roumaine. C'est ce qui nous permet d'anticiper le désir de langue roumaine, propre à Lastotchka, le personnage de Tatiana Țîbuleac - certes un désir d'amour, mais un amour impossible. De plus, quand Radu, le jeune professeur de roumain lui reproche de tordre la syntaxe de la langue, on entend en écho, dans le ressenti de la jeune fille hantée par les deux langues, les complaintes du "métèque » Cioran :

«Si j'avais été Français, je crois que je n'aurais prêté aucune attention à l'écriture. Mais c'est le drame du métèque, de songer sans cesse qu'il manie une langue qui n'est pas la sienne. Ensuite, vivre dans un pays où le tour de phrase compte, n'arrange pas les choses. » (Cioran, 1997 : 662)

\section{Contexte géopolitique et diglossie}

L'univers du roman projette le lecteur dans la capitale de la République soviétique de Moldavie, le Chișinău des années 1980-1990, décennie qui précède son indépendance. Dans la cour d'immeuble d'un quartier populaire, des bribes de récits de vie, des personnages hauts en couleur et un voisinage cosmopolite forment un vivre-ensemble pittoresque et attachant qui passe par l'usage de deux idiomes, le russe et le roumain, dans une situation de diglossie historique. Nous choisissons d'employer le terme "diglossie » et non celui de bilinguisme, dans l'acception que lui donne Rainier Grutman (2003) :

"Par "diglossie", on entend tantôt la répartition fonctionnelle de deux variétés linguistiques dans une société donnée, tantôt leur superposition conflictuelle. Selon les uns, dont le propagateur du terme, Charles Ferguson (1959), la diglossie serait un modus vivendi plutôt stable, tandis que d'autres estiment qu'elle favorise les tendances assimilatrices.

La répartition, en effet, ne se fait pas de manière aléatoire mais va souvent de pair avec une distribution sociale des valeurs accordées aux langues respectives. Se développe alors un parallélisme entre une fraction de la société et sa pratique langagière, de telle sorte que la diglossie peut devenir une source de conflits.

$[\ldots]$ Les langues entre lesquelles le sujet diglossique $[\ldots]$ croit avoir le choix ne sont pas de simples outils de communication, mais des formes d'expression indexées à des valeurs symboliques. » (Grutman, $2003: 118)$

Dans Le Jardin de verre, monde fictionnel, cadre géopolitique et langue d'écriture dessinent de concert une mise en abîme symbolique : 
l'auteur décrit, en roumain, le combat avec le russe d'une orpheline moldave qui tente de se construire entre les deux langues.

Pour situer le cadre géopolitique, il faut rappeler que le démantèlement de l'URSS au début des années 1990 a laissé à la frontière de l'Europe quelques pays en quête de liberté et d'identité : c'est le cas de la République de Moldavie, petit pays de l'Europe orientale dont les quelque trois millions d'habitants parlent le roumain - la langue officielle, mais aussi le russe et l'ukrainien. Roumaine par le fait d'un héritage historique de plusieurs siècles, cédée toutefois à l'Empire russe en 1812, la République de la Moldavie, appelée également Bessarabie, s'unit au Royaume de Roumanie en 1918. Mais, à la fin de la Deuxième Guerre mondiale, la Bessarabie est intégrée à l'Union Soviétique jusqu'à ce que ce petit pays proclame son indépendance en 1991. Tiraillée, au gré de l'Histoire, entre deux sphères d'influence voisines que tout oppose - l'une latine, l'autre slave - la République de la Moldavie développe une culture hybride basée sur la cohabitation tendue au quotidien entre roumanophones et russophones, tous censés incarner en premier l'identité et l'authenticité de ce peuple de l'Est.

Plusieurs écrivains moldaves d'aujourd'hui (Iulian Ciocan, né en 1968, Savatie Baștovoi, né en 1976) s'emparent de cette situation de diglossie et de tension identitaire pour en faire des ingrédients romanesques (Baștovoi, 2018, Ciocan, 2017) et parler d'une société moldave postsoviétique, certes libre, mais aussi en proie à la pauvreté, à la corruption et à la décadence morale. Quant à Tatiana Țîbuleac (née en 1978), elle réussit dans ce deuxième roman ${ }^{1}$ à rendre encore plus sensible la problématique de la diglossie, dans la mesure où le conflit entre les langues, le dilemme de l'entre-deux, constitue la substance même de la diégèse, la trame qui porte le devenir-sujet du personnage principal.

\section{Diglossie dans le texte littéraire}

Au niveau graphique, le roman de Tatiana Țîbuleac est également un montage diglossique, la graphie en caractères latins, ceux du roumain, étant émaillée de mots ou d'expressions en lettres cyrilliques, celles du russe. Le roumain du texte est parasité, presque rongé par le russe, car chaque langue garde son alphabet et donc sa marque identitaire, irréconciliable visuellement. À la différence d'un texte qui imagine un lecteur aux compétences bilingues, le texte en situation de diglossie opère par redondance sémantique :

\footnotetext{
${ }^{1}$ Le premier roman de Tatiana Țîbuleac, Vara în care mama a avut ochii verzi [L'été où maman a eu les yeux verts], a été publié en 2017 aux éditions Cartier de Chișinău. Les deux romans sont traduits en français par Philippe Loubière et publiés aux éditions des Syrtes, en 2018 et en 2020. Tous les extraits du Jardin de verre cités dans le présent article proviennent de l'édition française mentionnée.
} 
«Le texte diglossique aussi se distingue du texte bilingue. Dans ce dernier, strictement parlant, l'irruption de la langue étrangère n'est pas encadrée, amortie, neutralisée par une batterie de traductions ou de paraphrases. Il n'y a pas de redondance sémantique entre des codes linguistiques qui se complètent et font donc implicitement appel à un lecteur bilingue. Dans les textes diglossiques, par contre, la coprésence des langues fonctionne différemment parce qu'elle repose sur une certaine redondance. » (Grutman, $2003: 8$ )

Chez Tatiana Țîbuleac, les termes russes, opaques pour un nonbilingue, sont systématiquement traduits dans des notes de bas de page, de sorte que la lecture d'un unilingue n'est affectée que par un simple harcèlement linguistique, légitimé d'ailleurs par la portée symbolique du récit. Le contraste entre les deux alphabets, graphiquement contrariant dans une même phrase, renvoie subtilement à l'exercice quotidien d'une forme de schizophrénie linguistique, psychologique et culturelle, propre à l'univers de la diégèse.

\section{Le désir de langue et sa po(i)étique}

Patchwork elliptique et composite de tableaux courts et imagés, la narration est prise en charge par deux voix qui se juxtaposent et se suivent, celle de la maturité et celle de l'enfance. Une mère célibataire, aujourd'hui médecin en Roumanie, se rappelle la fillette qu'elle a été à Chișinău, dans les années 1980. Son destin âpre d'enfant exploitée par une mère adoptive, son devenir-sujet passe par un processus de subjectivation où la soumission contrariante et contrariée au prestige de la langue russe d'abord et au désir de langue roumaine ensuite constitue l'intrigue de l'histoire. L'incipit donne une voix à la fillette qui a la conscience poétique de sa re-naissance, sa deuxième venue au monde: adoptée par une ramasseuse de bouteilles, l'orpheline moldave découvre la ville et la langue russe.

«Je nais la nuit, j'ai sept ans. Elle me prendrait bien dans les bras, me dit-elle, mais elle a les mains occupées. Une lampe bleue, attachée à un arbre avec un câble, éclaire de haut. Elle se balance. Je tourne la tête en arrière et je la vois mieux : elle est ronde, comme un pain entier. Nous passons les portes de la ville comme on entrerait dans un ventre de pierre. En ville, c'est comme cela, pensé-je. Encore et toujours en pente, le chemin. La glace nous colle aux talons, la rue devient plus courte. Elle me tend sa poche pour que je m'y accroche et ne glisse pas. Que je regarde autour de moi, et que je voie de la beauté, moi aussi ! Cette lumière tamisée. Ce ciel aux étoiles en fuite. Des immeubles, des immeubles, des immeubles. Aucun n'a plus de quatre étages. Aucun ne dépasse quatre fenêtres en largeur. Sa poche a de la fourrure, mes ongles deviennent feu. Par les fenêtres, on distingue des gens de petite 
taille qui vivent bien. Des milliers de carrés avec une flamme au milieu. Les uns à côté des autres, les uns au-dessus des autres. Ceux d'en bas tiennent les autres par les épaules. Ceux d'en bas sont costauds. Un chien - bleu - marche derrière nous, en laissant ses petites empreintes. Je me dis qu'en ville, tout est bleu et va par quatre. Je ne dois pas rester en arrière, je ne dois jamais rester en arrière. Nous nous arrêtons à côté d'une clôture. Закрой глаза и забудь всё* [traduction dans la note en bas de la page : "Ferme les yeux et oublie tout."]. Je ne comprends rien, j'oublie tout en une seconde. » (Țîbuleac, $2020: 9-10$ )

En position d'émerveillement, le regard poétique de cette fillette malheureuse qui, à la place des mots, voit des épines, à la place des gens voit des arbres, transfigure et trouve une forme de beauté (un style) à sa quête éprouvante d'identité. L'émerveillement est le mode opératoire, la poïétique qui fait avancer la matière romanesque.

« À Chișinău, il y a une rue, la rue la plus longue et la plus difficile du monde. Dans cette rue, les bâtiments, les arbres, les feux rouges, y compris les poubelles et les fondrières, tout parle le russe. Une seule fois, elle m'a dit que plutôt que de parler russe comme une plouc, il valait mieux que je parle moldave. Русский язык не вто-ро-го сорта* [traduction dans la note en bas de la page : "le russe n'est pas une langue de seconde catégorie"]. Elle a plié son index en triangle et m'a frappé sept fois sur le front. Pourquoi sept fois, comment sept fois ? Une fois pour chaque mot et trois fois pour вто-ро-го*, par syllabes. Si elle me les avait marqués au fer rouge sur le front, elle m'aurait fait moins mal. » (T,îbuleac, 2020 : 25-26)

Tamara Pavlovna, la maman adoptive de Lastotchka, surnom de l'orpheline, lui intime d'apprendre le russe, de parler « en langage humain » (T,îbuleac, 2020 : 58). L'expression brute, en lettres cyrilliques sur la page, rappelle en écho la pensée de la philosophe Barbara Cassin qui, en faisant l'étymologie grecque de son prénom, dénonce la prétention hégémonique de se croire en possession d'une langue universelle et de désigner les locuteurs d'une autre langue comme des barbares:

« Les barbares, ceux qui disent „,bla bla bla”. Ceux qu’on ne comprend pas quand ils parlent: une onomatopée pour dire qu'ils font du bruit. Comme „berbère”, „babil”, Babel, ou „borborygme”. Un mot passionnant, puisqu'il dit: voilà, il y a quelque chose qu'on ne comprend pas, c'est de l'ordre du signifiant et on ne sait pas quoi en faire. Pour les Grecs, les Grecs du logos, ces barbares-là, ce sont les autres. » (Cassin, $2020: 69-70)$ 
Lastotchka dépasse sa condition de barbare au prix d'une souffrance qu'elle s'inflige pour ne pas perdre le lien affectif qui la lie à la mère adoptive. La mal aimée, la Moldave orpheline est en manque d'amour, et conquérir l'amour de l'autre prend la forme d'une torture linguistique. Sur le plan stylistique, corps et verbe forment l'isotopie de la scène, le corps est verbe.

«Le russe était devenu un visage toujours froncé. Beau, surnaturellement beau, cependant plein de cruauté. Quand il me souriait, même les épines devenaient fleurs, autour de moi. Mais quand je me trompais... La reine de glace que j'étais redevenait une fille de l'orphelinat avec laquelle je commençais à me crêper le chignon. Je pleurais chaque jour. Je poussais les mots du pied, pour les faire sortir de moi. Je les retirais de ma chair avec les dents, comme des épines d'églantier. Devant les difficultés, je m'enfonçais les ongles dans les cuisses. De cette façon, la douleur se ressentait dans plusieurs endroits à la fois. Les sons devenaient plus limpides et sortaient de moi clairs et corrects. Même comme cela, изящно* [traduction dans la note en bas de la page : "gracieux"], je l'ai répété quatorze fois et j'ai reçu quatorze coups de son index en triangle sur le front. Pour скороход* [traduction dans la note en bas de la page : "personne où voiture qui va très vite"], neuf coups. » (T,îbuleac, $2020: 32$ )

Bien que «plus russe qu'au moment de [sa] naissance », pourtant, le désir de l'autre langue - le moldave - surgit de nulle part et la fillette affronte sa mère en s'opposant à ce qu'elle aille poursuivre sa scolarité à l'école russe : " J'ai choisi les Moldaves, j'ai choisi la lie. Elle m'a battu jusqu'à ce que les bras lui en tombent. Une chienne qui mordait la main qui la nourrissait, voilà ce que j'étais. "Demandons conseil à Bella" a-t-elle fini par dire. » (Țîbuleac, 2020 : 76). Entre Lastotchka et Bella - la sage voisine auprès de laquelle la marâtre déçue cherche conseil - a lieu une discussion secrète. Cet indicible mystérieux, que l'auteur tait dans la diégèse, est symbolique : la raison profonde nous résiste, le pourquoi ontologique d'une langue ne se dit pas, le fantasme est aveugle : est-ce la langue qui nous choisit ? ne la choisiton pas?

Le retournement contre le russe puis le retournement contre le roumain font partie du devenir-sujet du personnage et le désir de langue se fait désir d'homme. Mais Radu, le professeur de roumain qui lui donne des cours particuliers, ne voit pas que son élève se mue en femme. Le désir se fait amour-haine, désenchantement de la langue.

« Je continuais à me rendre chez Radu deux fois par semaine. Entre nous, il ne se passa rien de ce que j'aurais voulu, mais j'obtenais au moins de bonnes notes en roumain. En grammaire, je me 
débrouillais mieux qu'à l'oral, où j'avais encore des problèmes avec les terminaisons. Je mettais les mots à l'envers. Un jour, il m'a offert un café et il m'a priée de ne plus du tout parler le russe. De m'enlever le russe de la tête, de l'enlever de partout, de sentir la beauté de ma langue. Cela m'a fait sortir de mes gonds, même si ce n'était pas le problème. Radu me regardait comme on regarde un enfant. J'aurais désiré qu'il m'apportât des fleurs, et lui me gavait de biscuits. Je m'habillais de robes légères, et lui fermait la fenêtre pour que je n'attrape pas froid. Je l'aurais tué. Lui sauter dessus, je ne pouvais pas, et je n'aurais même pas su que faire ensuite. Mais pour lui dire que je l'aimais, mes dents ne se desserraient pas. » (Țîbuleac, 2020 : 237)

Cet amour trouble et contrarié se matérialise sur la page par des signifiants étrangers et hybrides, en cyrillique et en lettres latines, portant dans leur cœur le nom du désiré. en tête.

« Je ne pensais qu'à Radu, dans toutes les langues que j'avais

\author{
Raduга, \\ Raduюcь, \\ Kraduщийся, \\ Raduшный*1 \\ Bradul - ёлка !2 \\ [1*Respectivement : "Arc-en-ciel,/ Je me réjouis,/ Qui se
} faufile,/ Amical. » 2. En roumain puis en russe : « sapin ».] 》 (Țîbuleac, $2020: 218)$

Sur le même plan - la forme de la substance (Hjelmslev 1984, 2000) , il faut observer que la narratrice se raconte constamment dans une syntaxe inversée. Ce que Radu, le professeur de roumain, lui reproche assez tardivement dans la diégèse [ " Tu mets encore les mots à l'envers » (Țîbuleac, 2020 : 234), en lui intimant de ne plus inverser les mots et d'oublier le russe, représente de fait une constante stylistique de l'écriture du roman. Dans de nombreuses occurrences, le complément d'objet direct où l'attribut du sujet sont rangés en début de phrase, ce qui, sur le plan de la substance de la forme, renvoie symboliquement à la perception constamment décalée de la fillette, cet entre-deux, deux langues, deux pays, deux cultures. De cet entredeux Lastotchka fait son espace de liberté. Devenue trait stylistique dans le roman de Tatiana Țîbuleac, la malléabilité syntaxique récurrente, presque incantatoire par effet d'accumulation, sert la poïétique de l'entre-deux.

L'idylle manquée entre Lastotchka et Radu porte en creux un autre implicite douloureux, le constat d'un amour impossible. Le plan poétique rejoint ici le plan politique, le désir de langue irrigue le désir de Roumanie, mais le rendez-vous est-il possible, la Roumanie sera-t-elle au rendez-vous ? 
La nostalgie du paradis perdu est la dimension proprement politique du roman. Pour l'orpheline moldave - de fait, abandonnée par ses parents roumains à un orphelinat - le terminus paradis est la Roumanie. Quel goût a ce retour au paradis pour l'adolescente qui devient étudiante en médecine en Roumanie et finalement docteur à Bucarest? La réponse implicite réside peut-être dans ce filon mélodramatique de l'histoire qui veut que Lastotchka devienne maman d'une petite fille atteinte de la maladie dite « des os de verre » dont elle s'occupe seule, après la désertion de son mari devant la souffrance et la fragilité de l'enfant. Une course d'obstacles placée en pleine maturité et en territoire roumain. Une fois de plus un rendez-vous manqué, la Roumanie n'a pas été à la hauteur.

\section{En guise de conclusion}

Dans le monde fictionnel imaginé par la romancière Tatiana Țîbuleac, la petite Lastotchka ambitionne d'apprendre le russe mais choisit le roumain comme langue d'étude et de maturité professionnelle et affective. Le personnage évolue entre l'injonction orgueilleuse de parler «la langue humaine ", suprême, d'après sa mère adoptive, et l'orgueil d'avoir trouvé sa propre forme de suprématie, le droit à son propre choix.

À travers la poïétique de l'entre, analysée tant au niveau stylistique qu'au niveau symbolique, c'est la possibilité de s'affranchir de l'emprise d'une langue, d'un pays, d'une culture - et finalement de tout déterminisme que le roman affirme par les deux voix intercalées. Celle de la fillette au regard émerveillé qui ne rêve que d'atteindre le paradis voisin, et celle de la maman désenchantée qui de sa course d'obstacles a pourtant tiré et intériorisé une forme poétique de sagesse et de beauté. Si la poétique est aussi politique, chez Tatiana Tî̉buleac, entre la Moldavie et la Roumanie, l'idylle reste toujours entre.

\section{Références :}

Cioran, E. (1997). Cahiers 1957-1972/ Notebooks 1957-1972. Paris : Gallimard.

Cioran, E. (2011). Euvres/ Complete works. Paris : Gallimard.

Deleuze, G; Guattari, F. (1980). Mille plateaux/ A Thousand Plateaus. Paris : Minuit.

Rainier, G. (2003). Bilinguisme et diglossie: comment penser la différence linguistique dans les littératures francophones/ Bilingualism and diglossia: how to think about the linguistic difference in French-speaking literatures. In Lieven D'hulst et Jean-Marc Moura, (dir.) Les études littéraires francophones : état des lieux. Lille: Presses Universitaires de Lille, 113126. 
Baștovoi, S. (2018). Les Enseignements d'une ex-prostituée à son fils handicapél The Teachings of an Ex-Prostitute to Her Disabled Son. Paris : Jacqueline Chambon/Actes Sud.

Casanova, P. (2015). La Langue mondiale. Traduction et domination/ The World language. Translation and domination. Paris : Seuil, Coll. Liber.

Cassin, B. (2016). Eloge de la traduction. Compliquer l'universel/ Praise of translation. Complicating the universal. Paris : Fayard.

Cassin, B. (2020). Le bonheur, sa dent douce à la mort. Autobiographie philosophiquel Happiness, her sweet tooth to death. Philosophical autobiography. Paris : Fayard.

Ciocan, I. (2017). Le Royaume de Sasha Kozak/ Sasha Kozak's Kingdom. Paris : Belleville.

Deleuze, G. (1969). Logique du sens/ Logic of Sense. Paris : Minuit.

Derrida, J. (2005). Apprendre à vivre enfin. Entretien avec Jean Birnbaum/ Learning to live. A conversation with Jean Birnbaum. Paris : Galilée.

Hjelmslev, L. (2000). Prolégomènes à une théorie du langage : La Structure fondamentale du langagel Prolegomena to a Theory of Language: The Basic Structure of Language. Paris : Minuit.

Hjelmslev, L. (1984). Le langage : une introduction/Language : An Introduction. Paris : Minuit.

Meschonnic, H. (1999). Poétique du traduirel Poetics of translating. Lagrasse: Verdier.

Prieur, J.-M. (2006). Contact de langues et positions subjectives/ Languages. Contacts and Subjective Positions. In Langage et société 2006/ 2, n ${ }^{\circ} 116$, 11-118. https://doi.org/10.3917/ls.116.0111 consulté le 20.01.2021

Rivarol (1998). Discours sur l'universalité de la langue française/ Speech on the universality of the French language. In Pensées diverses. Verrièrres-leBuisson : Desjonquères, 102-157.

Țîbuleac, T. (2017). Vara în care mama a avut ochii verzil The Summer when Mother had Green Eyes. Chișinău: Cartier.

Țîbuleac, T. (2018). L'été où maman a eu les yeux verts. Genève : Syrtes.

T,îbuleac, T. (2018). Grădina de sticlă/ The Glass Garden. Chișinău: Cartier.

Țîbuleac, T. (2020). Le jardin de verre/ The Glass Garden. Genève : Syrtes.

Wismann, H. (2012). Penser entre les langues/ Think Between Languages. Paris : Albin Michel. 